\title{
Acute Stroke Multimodal Imaging: Present and Potential Applications toward Advancing Care
}

\author{
I. Matos Diaz, $\mathrm{MD}^{1, *} \quad$ J. W. Liang, $\mathrm{MD}^{2,3, *}$
}

L. Velickovic Ostojic, MD ${ }^{3}$ Aaron P. Tansy, MD ${ }^{1}$

Address for correspondence Aaron P. Tansy, MD, Department of Neurology, Mount Sinai Comprehensive Stroke Center, One Gustave L. Levy Place, Box 1052, New York, NY 10029 (e-mail: neuroectomy@gmail.com).

\author{
Abstract \\ Keywords \\ - acute ischemic stroke \\ - stroke \\ - imaging \\ - perfusion
}

In the past few decades, the field of acute ischemic stroke (AIS) has experienced significant advances in clinical practice. A core driver of this success has been the utilization of acute stroke imaging with an increasing focus on advanced methods including multimodal imaging. Such imaging techniques not only provide a richer understanding of AIS in vivo, but also, in doing so, provide better informed clinical assessments in management and treatment toward achieving best outcomes. As a result, advanced stroke imaging methods are now a mainstay of routine AIS practice that reflect best practice delivery of care. Furthermore, these imaging methods hold great potential to continue to advance the understanding of AIS and its care in the future.
Over the past few decades, the field of acute ischemic stroke (AIS) has made landmark strides in routine care. Pivotal to this advancement has been the incorporation and utilization of advanced neuroimaging, which has afforded stroke care providers a significant improvement in understanding of AIS pathophysiology and identification of its subclassifications toward better informed therapeutic decision making and resultant outcomes. As a consequence, acute stroke multimodal imaging methods are now recognized as an essential component of empirically based, best practice delivery of AIS care. ${ }^{1,2}$ Furthermore, because of its now firmly established impact on and foothold within AIS management, advanced stroke imaging has positioned itself as a continued prime player in the field's ongoing goal to reduce barriers to treatment and facilitate success in outcomes.

In this review, we address the powerful ability of advanced acute stroke imaging to rapidly and accurately profile, understand, and characterize AIS pathophysiology, its crucial role in AIS routine practice currently, and the poten-

\footnotetext{
Co-First authors.
}

tial for its novel applications to continue to shape and advance the field of AIS in the future.

\section{Pathophysiology of AIS}

An understanding of cerebrovascular hemodynamics and stroke pathophysiology is essential to the interpretation and application of information that acute stroke imaging provides. Cerebral perfusion pressure (CPP) is equal to mean arterial pressure (MAP) minus intracranial pressure (ICP, Eq. 1). In ischemic stroke, sustained increases in ICP (from cytotoxic edema) or sudden drops in MAP (from arterial occlusion) can lead to a reduction in CPP. The protective mechanism of cerebrovascular autoregulation helps maintain a constant cerebral blood flow (CBF) over a wide CPP range by altering the caliber of cerebral arterioles ( $\mathbf{- F i g . 1}$ ). Thus, CBF is defined as CPP divided by cerebrovascular resistance (CVR, Eq. 2).

Eq. 1: $C P P=M A P-I C P$

Eq. 2: $C B F=C P P / C V R$

Copyright (c) 2017 by Thieme Medical Publishers, Inc., 333 Seventh Avenue, New York, NY 10001, USA. Tel: +1(212) 584-4662.
Dol https://doi.org/ 10.1055/s-0037-1607355. ISSN 0271-8235.
Editor, Joshua P. Klein, MD, PhD, FANA FASN, FAAN 


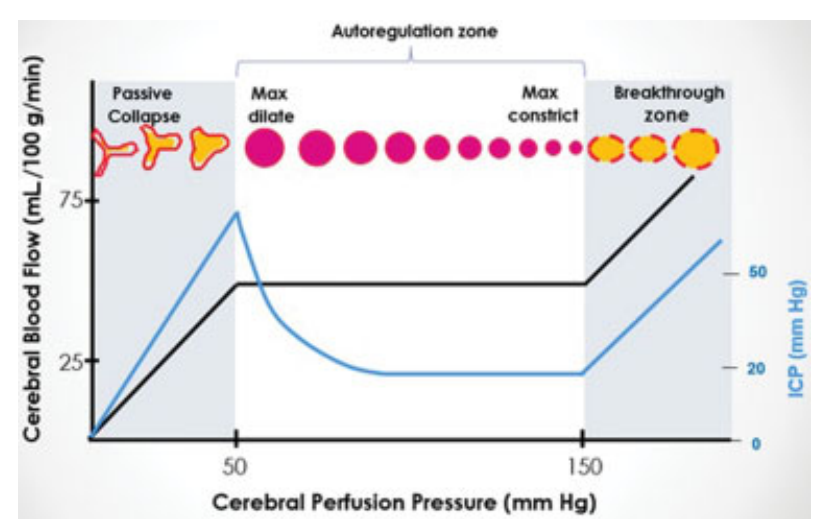

Fig. 1 Cerebrovascular autoregulation: relationships between vascular caliber and intracranial pressure on cerebral perfusion pressure and cerebral blood flow (Adapted from Rose JC, Mayer SA. Optimizing blood pressure in neurological emergencies. Neurocrit Care 2006;4(1):98).

AIS has historically been classified into three distinct stages of cerebral hemodynamics. In stage I of an ischemic event, the cerebrovascular system labors to maintain a constant CBF in the face of low CPP through vasodilation of cerebral arterioles and recruitment of collateral cerebrovascular supply. At this stage, despite stress on the affected cerebrovascular territory, compromise and resultant ischemia of its supplied territory is avoided, theoretically, indefinitely. However, as the limits of autoregulation are reached and CBF nears the critical ischemic threshold, the cerebrovascular system enters stage II of AIS in which hemodynamic failure and increase of oxygen extraction occur. During this stage, the cerebral tissue is at risk of infarction, if reperfusion is not achieved promptly. This area of potentially salvageable tissue is termed the ischemic penumbra. With further reductions in CBF, failure of the collateral cerebrovascular supply follows, causing oxygen extraction to wane and become no longer sufficient to maintain tissue metabolism. Thus, irreversible injury and cell death (ischemic core) result.

Although AIS revascularization therapies have been well established to improve outcomes on a population basis, a significant proportion of patients treated within established timeframes of eligibility, unfortunately, do not reap significant functional improvement. ${ }^{3}$ On a patient level, these effects are likely attenuated and/or negated because these time windows of symptom duration do not adequately account for variations in individual cerebrovascular anatomy, cerebral autoregulation reserve, and collateral cerebrovascular supply, and status. Cerebral collateral circulation helps stem expansion of infarct core volume via shunting the blood supply around an arterial occlusion through alternative arterial pathways, thereby maintaining adequate CBF to the affected field of tissue. Stroke severity and rate of ischemic injury progression are largely dependent upon a complex interplay between, among others, robustness of collateral circulation, the CBF nadir, and chronic disease states, such as chronic hypertension, which can shift the limits of autoregulation. ${ }^{4}$ For example, for a given affected territory, a patient with low CBF and poor baseline collateral anatomy and/or status is likely to more quickly "complete" conversion from penumbra to ischemic core than, for example, a patient with higher CBF and good baseline collateral anatomy and/or status. ${ }^{5}$

\section{Present Applications of Acute Stroke Imaging}

The goal of acute stroke imaging is to provide a rapid, accurate snapshot of acute brain and cerebrovascular pathology to determine which patients are most likely to be favorable candidates for and responders to revascularization therapies, such as intravenous (IV) tissue plasminogen activator (tPA) and endovascular revascularization therapies (ERTs). Brain imaging, traditionally and most commonly with noncontrast head CT, plays an essential role in AIS treatment selection and eligibility determination. For one, it is the only imaging modality required for determination of eligibility for IV tPA administration. Its main role is to exclude radiographic contraindications to IV tPA, namely, intracranial hemorrhage, large ischemic strokes (less than one-third of the middle cerebral artery [MCA] territory), and structural lesions such as tumors. ${ }^{6,7}$ Despite the fact that CT has low sensitivity (42-63\%) for detection of acute and small ischemic infarcts, especially in the posterior fossa, ${ }^{8}$ its rapid acquisition time, relative ease of identification of hemorrhage by nonradiologists, and widespread availability in the community make CT the recommended imaging modality in the time-sensitive evaluation for IV tPA. ${ }^{7}$

Even though CT is the clear modality of choice in routine practice, MRI is utilized at some centers as the first-line brain imaging method. MR imaging holds a few key advantages over CT-based brain imaging in AIS care. For one, diffusionweighted imaging (DWI) offers superior sensitivity and specificity over CT in AIS detection. DWI abnormalities appear within minutes after ischemia occurs and may be detected in as many as 96.1 to $99.6 \%$ of patients when performed within 6 hours of the onset. ${ }^{9}$ Moreover, through multiple different imaging sequences (fluid attenuated inversion recovery [FLAIR], gradient echo [GRE], and T2-weighted sequences), ${ }^{10}$ MRI provides more information about ischemia-associated lesions that CT cannot offer, including high resolution and accuracy of location and pattern, chronicity, and ischemic/ hemorrhagic components, all of which lend additional diagnostic power toward assessment and understanding of lesion etiology. While MRI can offer enhanced diagnostic power to the stroke care provider and thus improved ability to guide AIS treatment, it has a few important disadvantages (in comparison to noncontrast $\mathrm{CT}$ ) that have limited its widespread adoption; mainly, its use is often considered more time and cost prohibitive. For one, MR facilities at most centers are not typically physically located near the emergency department (ED) triage area, thus delaying expedient completion. Additionally, costs associated with MR facility maintenance and operation often may prove unjustifiable for around-the-clock availability, especially in centers of care where MRI may not be commonly utilized, such as in rural communities or in areas of low-population density. Furthermore, unlike CT, MRI requires safety screening and patient consent, which may lend to 
additional impediments and delays to timely completion, especially in select AIS subpopulations (e.g., those with aphasia, neglect, or impaired consciousness). Lastly, although MRI holds greater diagnostic power than CT in AIS management, it requires greater knowledge and familiarity with a wider range of aforementioned imaging sequences, and thus its diagnostic application often necessitates input from radiologists.

In addition to evaluating for such exclusions to tPA, like acute hemorrhage, brain imaging also enables stroke care providers to predict safety and efficacy of AIS treatment at the patient level. For one, early infarct core size holds key prognostic value for treatment decision making. ${ }^{11}$ This has resulted in its increasingly more commonplace use in routine practice, including through incorporation of formal scoring metrics, such as the Alberta Stroke Program Early CT Score (ASPECTS). ASPECTS is an empirically validated, 10-point systematic method for evaluation of early ischemia within the anterior circulation on noncontrast brain CT (-Fig. 2). The parenchymal field subserved by the vascular territories of the anterior fossa is divided into 10 distinct regions, each of which is assessed and graded for early ischemic changes as defined by relative hypodensity. From this tabulation, a composite score from 10 (no ischemia) to 0 (ischemia of entire field) is generated that provides a rapid assessment of ischemic burden within the anterior vascular field, and consequently, prognosis for AIS treatment decision making through its identification of candidates most likely to respond clinically favorably (ASPECTS $\geq 6$ ) to or to suffer complications from revascularization therapies. ${ }^{1,12}$ In addition to its well-established value in AIS care, another key advantage of ASPECTS is that it requires only basic familiarity with CT interpretation and the ASPECTS scoring methodology. Therefore, its powerful utility and ease of use encourage its readied adoption and use throughout the stroke care community at specialized and unspecialized centers alike.
In addition to providing critical information about status of parenchymal tissue, noncontrast brain CT also holds diagnostic utility in evaluation of vessel pathology in acute stroke care: the hyperdense thrombosed large-vessel sign. Visible as increased density, most classically in linear form for an affected proximal MCA vessel, the sign is highly suggestive of a largevessel occlusion (LVO) within the proper clinical context of an acute stroke syndrome attributable to occlusion of that vessel. This sign is seen in approximately only one-third of angiographically confirmed LVOs. ${ }^{13,14}$ Therefore, although its detection may provide guidance for treatment decision making, its absence does not permit such guidance.

While brain imaging has been a mainstay of AIS care for over two decades, acute stroke multimodal imaging has materialized as a necessary component of routine care only in the past few years. The success of the multiple, recent ERT trials that relied on cerebrovascular angiographic imaging for determination of AIS treatment eligibility within expanded time windows legitimized the necessity and paved the way for this radical update of imaging used in routine care. Their landmark achievement firmly established that the incorporation of such imaging methods into AIS care provides not only a further enhanced profiling of acute stroke pathophysiology through rapid detection and assessment of thrombotic clot burden and location, ${ }^{1}$ the presence of other vascular lesions (e.g., cerebral aneurysms, vascular malformations), and collateral vasculature status, but also, as a result, a powerful imaging-based strategy of management that enhances eligibility for therapy as well as prognosis.

The cerebrovascular imaging modality of choice is CT angiography (CTA) because of its wide availability, rapid acquisition time, and high sensitivity and specificity for LVO detection. ${ }^{15}$ Of note, CTA carries a risk, although small and of unestablished significance, of contrast-related nephropathy, ${ }^{16-18}$ particularly among patients with pre-existing impairment of renal function.

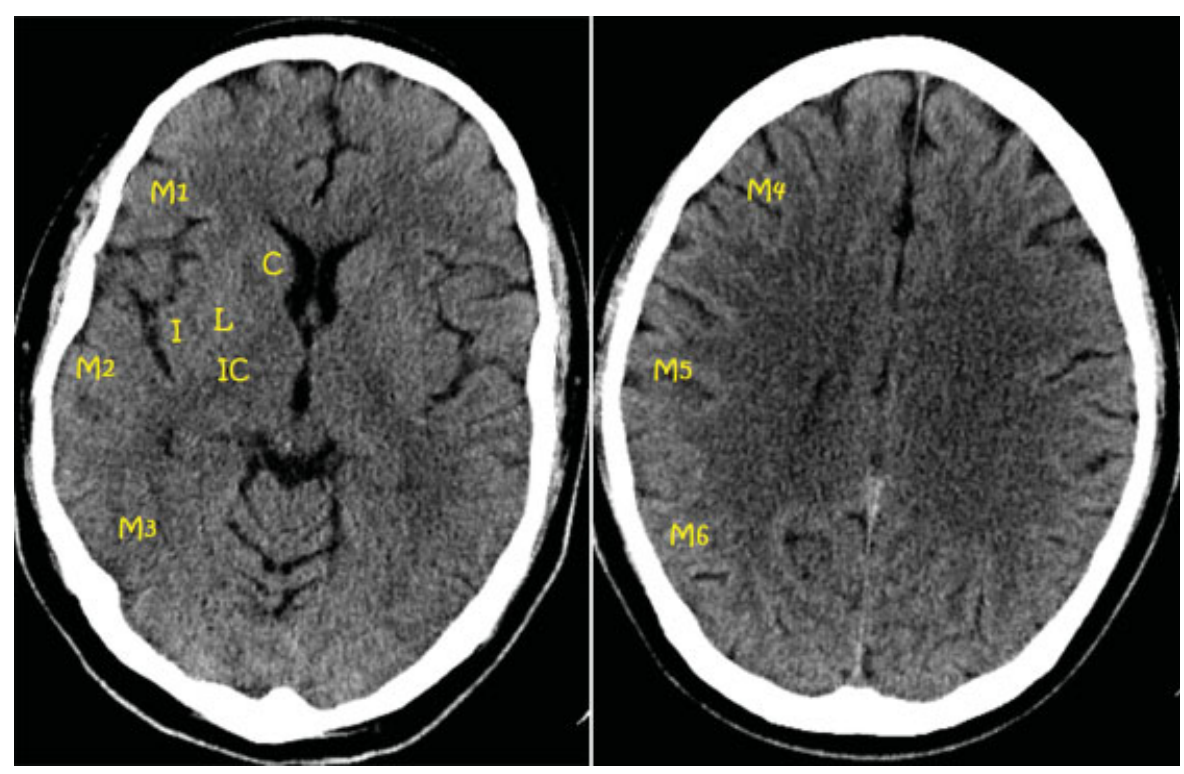

Fig. 2 ASPECT score. (A) Axial CT image at the level of basal ganglia and thalamus. (B) Axial CT image at supraganglionic level above the level of lateral ventricles. ASPECT, Alberta Stroke Program Early CT; CT, computed tomography. 
Magnetic resonance angiography (MRA) is another choice used as a first-line option at some centers; it has lower sensitivity but comparable specificity to CTA. ${ }^{15}$ Common MRA techniques include two-dimensional time of flight (TOF), three-dimensional TOF, and gadolinium-enhanced MRA. The gadoliniumenhanced technique allows improved visualization of distal vessels and is less susceptible to motion artifacts. The nonenhanced TOF, though more susceptible to motion artifact, may be preferred in patients with baseline kidney disease who are at risk for complications related to gadolinium exposure, including, most seriously, nephrogenic systemic fibrosis. While TOF MRA is helpful in identifying proximal LVOs, it is not considered as reliable for detection of distal vessel occlusions. ${ }^{19}$

The 2015 update of the American Heart Association (AHA)/ American Stroke Association (ASA) guidelines reflects the advent of advanced imaging as standard of care when considering endovascular treatment options for patients with AIS. ${ }^{1}$ Multimodal imaging, in the form of ASPECTS, as well as evidence of large vessel occlusion on CT angiogram, was a critical feature leading to the overall success of the endovascular trials (-Table 1). Despite some variation in selection criteria for the different trials, mainly regarding the minimum eligible National Institutes of Health Stroke scale (NIHSS) score and also the timeframe for inclusion, the use of more refined imaging tactics allowed for improved selection of patients who were more likely to have a beneficial response to endovascular intervention. The available data from the aforementioned studies led to the recommendation that endovascular intervention should be considered for patients who present with NIHSS and ASPECTS greater than 6 and an imaging-confirmed internal carotid artery (ICA) or MCA large-vessel (M1) occlusion within 6 hours from symptom onset.

In the wake of these recent advances and the AHA/ASA recommendations that were based upon them, treatment centers have begun to update their acute stroke imaging protocols to reflect these new standards in stroke care. As an illustrative example, a typical acute stroke imaging pathway for patients presenting to a comprehensive stroke center, with stroke symptoms suggestive of a LVO syndrome within 6 hours, is presented in -Fig. 4 and -Fig. 5.

\section{Future Applications of Acute Stroke Imaging}

The currently accepted model of AIS considers both the ischemic core and penumbra of an affected vascular territory as essential to the profile of AIS in vivo. Because it is at risk, but still viable tissue, the penumbra is the prime target of salvage for all revascularization therapies. ${ }^{20}$ Perfusion imaging modalities (CT and MR perfusion) allow estimation of penumbral volume through assessment of cerebral hemodynamics by tracking the flow of contrast within a region of interest and generating qualitatively informative maps for $C B F$, cerebral blood volume (CBV), mean transit time(MTT), and time to peak (TTP). These maps may, in turn, be used to estimate the volume of the ischemic core and ischemic penumbra (-Table 2 ).

Not surprisingly, because of its unique capability to provide penumbral status in AIS, perfusion-based imaging is considered to hold great potential in the continued advancement of AIS care. Integration of such advanced imaging methods into acute stroke imaging further refines the profile of AIS through an assessment that reflects, within an affected cerebrovascular territory, not only ischemic status (e.g., ASPECTS of noncontrast head (T) and thus infarcted tissue, which reduces candidacy for treatment, but also of penumbral status and thus tissue, which may respond best to it. As a result, perfusionbased acute stroke imaging theoretically allows the provider to depend less strictly, in absolute terms, on time/duration of symptoms: a potential key to further reduce time-based restrictions to improve outcomes from treatments. ${ }^{21}$ Although not yet evidence based, some stroke centers have already incorporated perfusion imaging into their acute stroke imaging protocol and may employ such a hypothetical pathway for

Table 1 Imaging criteria of the recent endovascular trials

\begin{tabular}{|c|c|c|c|c|c|c|}
\hline & Vessel imaging & Occluded vessel & Aspect & Perfusion imaging & $\begin{array}{l}\text { Symptoms } \\
\text { onset }\end{array}$ & Outcome $^{a}$ \\
\hline MR CLEAN & MRA, CTA, or DSA & $\begin{array}{l}\text { Distal ICA, M1, } \\
\mathrm{M} 2, \mathrm{~A} 1 \text {, or } \mathrm{A} 2\end{array}$ & Not used & Not used & $\leq 6 \mathrm{~h}$ & 32.6 vs. $19.1 \%$ \\
\hline EXTEND IA & MRA, CTA, or DSA & ICA, $M 1$, or $M 2$ & Not used & $\begin{array}{l}{ }^{\mathrm{b}} \text { Mismatch ratio } \geq 1.2 \text {, ischemic } \\
\text { core volume }<70 \mathrm{~mL}\end{array}$ & $\leq 6 \mathrm{~h}$ & 71 vs. $40 \%$ \\
\hline THRACE & CTA or MRA & $\begin{array}{l}\text { ICA, M1, or the superior } \\
1 / 3 \text { of the basilar artery }\end{array}$ & Not used & Not used & $\leq 5 \mathrm{~h}$ & 53 vs. $42 \%$ \\
\hline SWIFT PRIME & CTA or MRA & ICA, M1, or M2 & $\geq 6$ & $\begin{array}{l}\text { Mismatch ratio: } 1.2-1.8 \text { and } \\
\text { a small core of infarct }\end{array}$ & $\leq 6 \mathrm{~h}$ & 60 vs. $35 \%$ \\
\hline REVASCAT & CTA or MRA & ICA or M1 & $\begin{array}{l}\geq 7 \text { on } \mathrm{CT} \\
\geq 6 \text { on } \mathrm{DWI}\end{array}$ & Not used & $\leq 8 h$ & 43.7 vs. $28.2 \%$ \\
\hline ESCAPE & $\begin{array}{l}\text { CTA with scoring } \\
\text { of collaterals }{ }^{d}\end{array}$ & ICA, M1, or $\mathrm{M} 2$ & $\geq 6$ & Not used & $\leq 12 \mathrm{~h}$ & 53.0 vs. $29.3 \%$ \\
\hline
\end{tabular}

Abbreviations: CTA, computed tomography angiography; DSA, digital subtraction angiography; DWI, diffusion-weighted imaging; ICA, internal carotid artery; MRA, magnetic resonance angiography.

${ }^{a}$ Modified Rankin Score (0-2) at 90 days, treatment versus control groups.

${ }^{b}$ Perfusion assessed with an automated reading software (RAPID). Hypoperfusion was defined as $T_{\max }>6$ seconds. Ischemic core diagnosed as relatively $\mathrm{CBF}<30 \%$ of normal tissue or by DWI volume.

IInfarct less than one-third of the MCA territory on CT or DWI, or $>100 \mathrm{~mL}$ in other territories. Severe hypoperfusion $\left(\mathrm{T}_{\max }>10 \mathrm{~second}\right)<100 \mathrm{~mL}$.

${ }^{\mathrm{d}}$ Moderate-to-good collateral circulation was defined as the filling of $50 \%$ or more of the MCA by pial arterial circulation on CTA. 


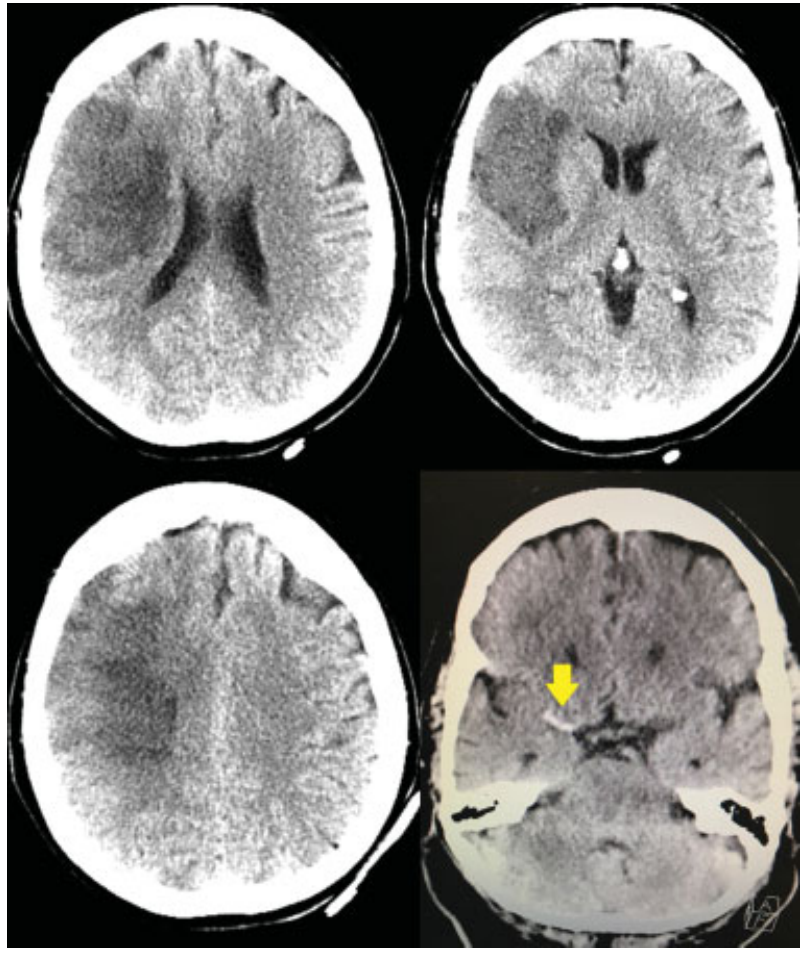

Fig. 3 A 70-year-old man with atrial fibrillation was brought to the ER with acute onset of left-sided weakness, left hemineglect, and a right gaze preference (NIHSS: 16). He was last seen well 5 hours prior to arrival to the ER. CT of the head showed a hyperdense right MCA vessel (arrow) and an ASPECTS of 5 (hypodensities in frontal, parietal, and insular regions). Given these findings, the patient was determined to be a poor candidate for acute treatment. ASPECTS, Alberta Stroke Program Early CT score; CT, computed tomography; ER, emergency room; MCA, middle cerebral artery; NIHSS, National Institutes of Health Stroke scale.

identifying AIS candidates for treatment who present beyond 6 hours from symptom onset (- Fig. $\mathbf{5}$ ).

In line with this rationale of use by some centers, the ability to translate perfusion-based imaging's unique assessment of salvageable tissue status into improved outcomes is currently

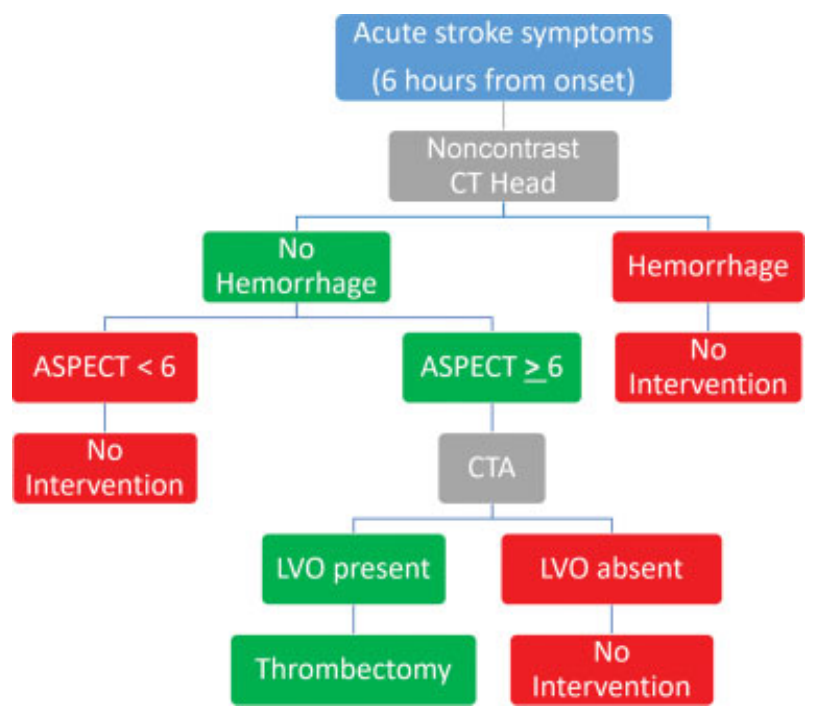

Fig. 4 Acute stroke imaging pathway for evaluation of patients presenting within 6 hours of symptom onset.
Table 2 CT perfusion definitions

\begin{tabular}{|l|l|l|l|}
\hline & CBV & CBF & MTT \\
\hline Definition & $\begin{array}{l}\text { Volume of blood } \\
\text { present in brain } \\
\text { parenchyma }\end{array}$ & $\begin{array}{l}\text { Rate of } \\
\text { blood flow } \\
\text { through brain } \\
\text { parenchyma }\end{array}$ & $\begin{array}{l}\text { Time needed for } \\
\text { blood to transit } \\
\text { through brain } \\
\text { parenchyma }\end{array}$ \\
\hline Core & $\downarrow$ & $\downarrow \downarrow$ & $\uparrow$ \\
\hline Penumbra & $\leftrightarrow / \uparrow$ & $\downarrow$ & $\uparrow$ \\
\hline
\end{tabular}

Abbreviations: CBF, cerebral blood flow; CBV, cerebral blood volume; $\mathrm{CT}$, computed tomography; MTT, mean transit time.

under investigation. ${ }^{22}$ Studies using perfusion-based techniques have already provided demonstration that profiles reflecting a small infarct core to large penumbra ratio are considered most likely to respond favorably to reperfusion therapies. ${ }^{23,24}$ The concept of perfusion-guided intervention in extended time windows is currently under investigation for both IV TPA and ERT ( - Fig. 6 and - Table 3). Both the European cooperative acute stroke study-4: Extending the time for thrombolysis in emergency neurological deficits (ECASS:4 ExTEND) $)^{25}$ and the multicenter, randomized, double-blinded, placebo-controlled Phase III study to investigate EXtending the time for Thrombolysis in Emergency Neurological Deficits (EXTEND) ${ }^{26}$ trials are testing the utility of perfusion imaging for an extended time window for IV tPA (up to 9 hours). Because it has been shown that ischemic penumbra may be present up to 24 hours in AIS, ${ }^{27}$ great interest has focused on expanding allowed time windows of treatment even further: the Endovascular therapy following imaging evaluation for ischemic stroke 3 (DEFUSE 3), ${ }^{22}$ Perfusion Imaging Selection Of Ischemic Stroke Patients For Endovascular Therapy (POSITIVE), ${ }^{28}$ and the Clinical Mismatch in the Triage of Wake Up and Late Presenting Strokes Undergoing Neurointervention With Trevo (DAWN) ${ }^{29}$ trials are evaluating the use of angiography (CT or MR based) in combination with

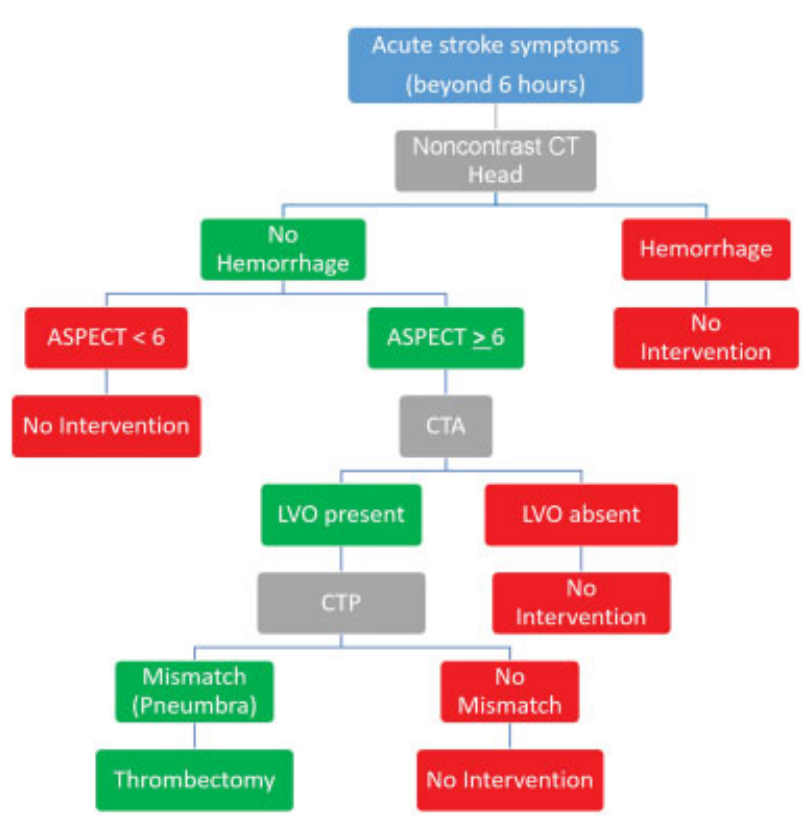

Fig. 5 Acute stroke imaging pathway integrating CT perfusion. CT, computed tomography. 


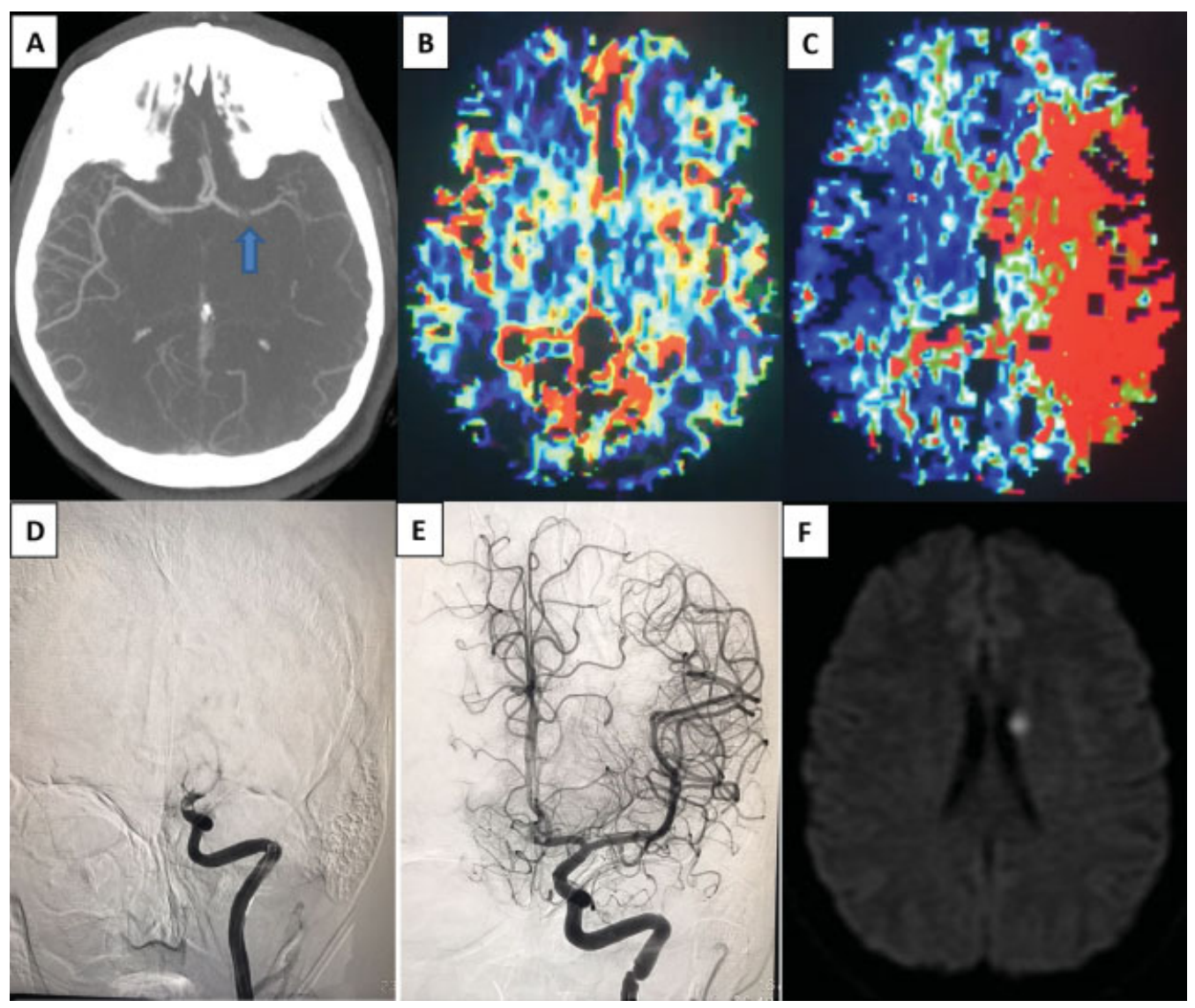

Fig. 6 A 54-year-old man presents with word-finding difficulty and left hemiparesis (NIHSS: 7) that was discovered upon awakening from sleep. He was last seen normal the night prior to presentation. CT of the head showed an ASPECTS of 10. (A) CT angiogram showed occlusion in left ICA terminus (blue arrow) with good collaterals. (B) Cerebral blood volume map showing no large ischemic core. (C) Increased mean transit time in left hemisphere suggestive of large mismatch/penumbra. (D) Conventional angiogram showing left ICA terminus occlusion. The patient was determined to be a favorable candidate for endovascular therapy and underwent revascularization with subsequent rapid clinical improvement. (E) Postthrombectomy with revascularization. (F) MRI brain DWI sequence 24 hours after thrombectomy showing a small periventricular infarct. ASPECTS, Alberta Stroke Program Early CT score; CT, computed tomography; DWI, diffusion-weighted imaging; ICA, internal carotid artery; MRI, magnetic resonance imaging; NIHSS, National Institutes of Health Stroke scale.

perfusion-based techniques for treatment up to 24 hours after symptom onset. Although inclusion criteria and perfusion parameters vary between these trials, a priori requisite assessment of a favorable penumbra/core volume ratio for determination of treatment candidacy is shared by all of them.
Although of currently unknown clinical utility, arterial spin labeled (ASL)-MRI is another emerging perfusion imaging method, which holds exciting implications for potential application in AIS. ${ }^{2,30}$ This technique assesses diffusion of arterial water to generate a CBF map, and thus estimate

Table 3 Ongoing trials using perfusion imaging to expand the treatment window

\begin{tabular}{|l|l|l|l|l|}
\hline & $\begin{array}{l}\text { Intervention } \\
\text { type }\end{array}$ & $\begin{array}{l}\text { Stroke onset/last } \\
\text { known normal }\end{array}$ & Imaging modalities & Imaging criteria \\
\hline $\begin{array}{l}\text { ECASS-4: } \\
\text { EXTEND }^{25}\end{array}$ & IV tPA & $4.5-9.0 \mathrm{~h}$ & MRI with PWI & $\begin{array}{l}\text { PWI:DWI ratio of } 1.2 \\
\text { Infarct core }<1 / 3 \text { of the } \mathrm{MCA} \\
\text { territory or }<100 \mathrm{~mL}\end{array}$ \\
\hline EXTEND $^{26}$ & IV tPA & $4.5-9.0 \mathrm{~h}$ & $\mathrm{CT}, \mathrm{CTP}, \mathrm{MRI}$ with PWI & PWI:DWI ratio of 1.2 Infarct core $<70 \mathrm{~mL}$ \\
\hline DEFUSE $^{22}$ & ERT & $6.0-16.0 \mathrm{~h}$ & $\mathrm{CT}, \mathrm{CTA}, \mathrm{CTP}, \mathrm{MRI}, \mathrm{MRA}, \mathrm{PWI}$ & ICA or M1 occlusion; target mismatch \\
\hline POSITIVE $^{28}$ & ERT & $6.0-12.0 \mathrm{~h}$ & $\mathrm{CT}, \mathrm{CTA}, \mathrm{CTP}, \mathrm{MRI}, \mathrm{MRA}, \mathrm{PWI}$ & ICA or M1 \\
\hline DAWN $^{29}$ & ERT & $6.0-24.0 \mathrm{~h}$ & $\mathrm{CT}, \mathrm{CTP}, \mathrm{MRI}, \mathrm{PWI}, \mathrm{CTA}, \mathrm{MRA}$ & $\begin{array}{l}\text { ICA or M1 occlusion, mismatch } \\
\text { on MR-DWI or CTP }\end{array}$ \\
\hline
\end{tabular}

Abbreviations: CT, computed tomography; CTA, CT angiography; CTP, computed tomography perfusion; ERT, endovascular revascularization therapy; IV tPA, intravenous recombinant plasminogen activator (ALTEPLASE); MRI, magnetic resonance imaging; PWI, perfusion-weighted imaging. 


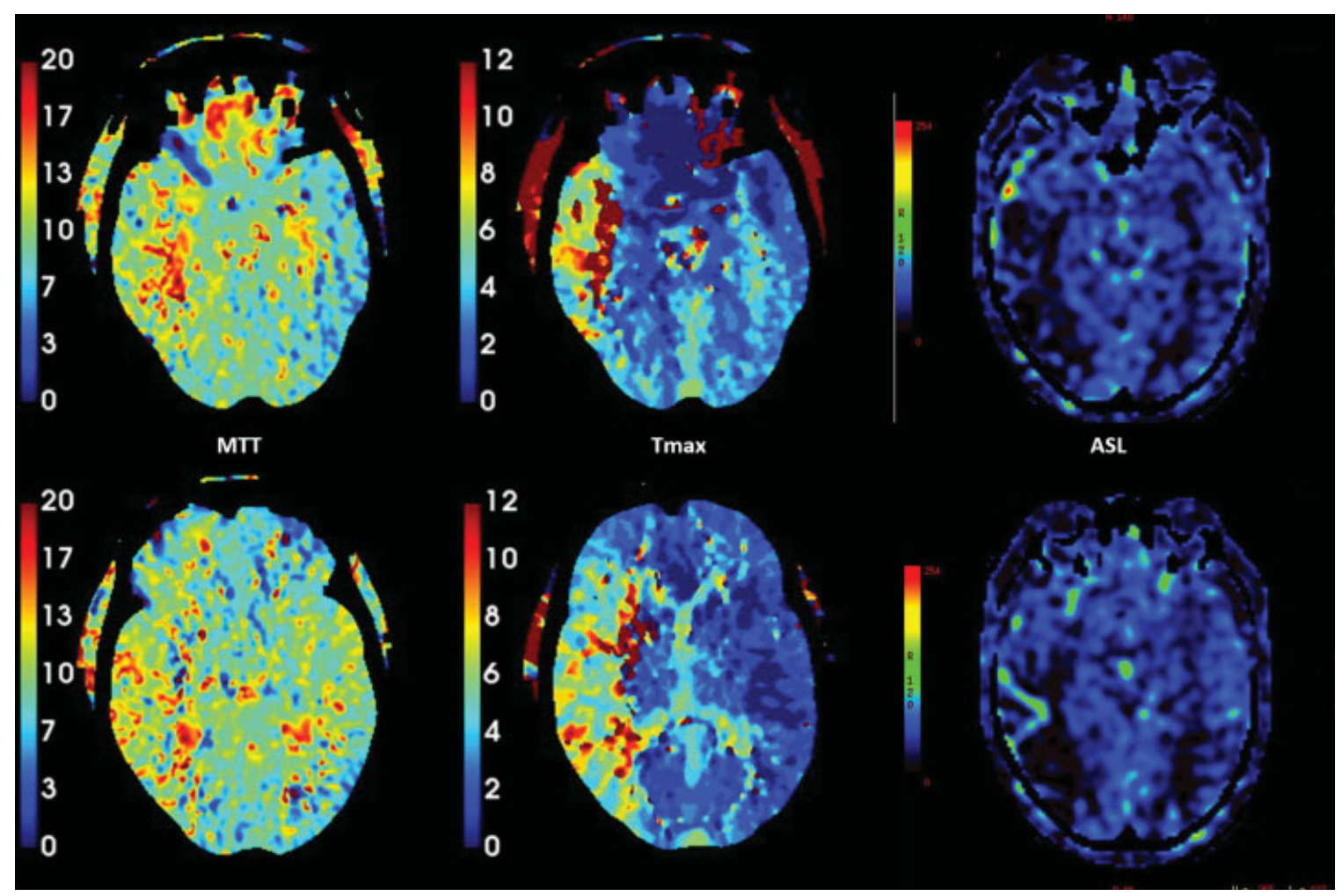

Fig. 7 ASL-MRI patient who presented with an acute right middle cerebral artery occlusion, out of the conventional 6-hour therapeutic window. CT perfusion (MTT and $\mathrm{T}_{\max }$ ) shows an area of hypoperfusion in the right hemisphere, which corresponds to the hypoperfused area on the ASLMRI done 24 hours later. ASL, arterial spin labeled; CT, computed tomography; MRI, magnetic resonance imaging; MTT, mean transit time; $\mathrm{T}_{\text {max }}$ time-to-maximum.

ischemic penumbra. A major advantage of ASL is that it requires no IV contrast, which makes its validation highly attractive as an imaging application that may enhance care without compromise of safety ( - Fig. 7).

As AIS investigations utilizing perfusion-based imaging continue to improve understanding of the ischemic penumbra and its relationship with and transition to the ischemic core, an improved and more accurate profiling of the AIS pathophysiology will hopefully follow toward achievement of ultimate goal of the stroke community: care freed from the restrictions of time and that optimizes chances for best outcomes.

\section{Transition from CT to MR Acute Stroke Imaging}

Although CT-based imaging has been the cornerstone of advanced imaging within acute stroke and its improvement in care, MR-based methods provide even greater promise for potential advances in AIS care. Not only are their sensitivity and specificity for detection of ischemia superior to CTbased methods, ${ }^{9}$ but also MR-based methods are acquired in equitably rapid fashion ${ }^{31}$ with reduced risk exposure. Hence, utilization of MR-based acute stroke imaging may prove to further AIS care in a variety of ways: refining diagnosis and thus increasing treatment rates, as well as mitigating unnecessary imaging and treatment exposure. For instance, implementation of rapid $\mathrm{MRI}^{31}$ protocols in routine AIS care may better identify ischemic events masquerading as nonischemic ones and exclude nonischemic ones mimicking ischemic ones.

\section{Conclusion}

Advanced stroke imaging has established itself as an integral component of AIS practice. Multimodal imaging methods afford an enriched understanding of acute stroke pathophysiology that empowers providers to deliver stroke care that reflects standards of care aimed at achievement of best outcomes. Advanced stroke imaging is likely to continue to be a driver of the ever-evolving field of AIS: one highlighted by less barriers to, and more successes from, treatment.

\section{Conflict of Interest}

None.

\section{References}

1 Powers WJ, Derdeyn CP, Biller J, et al; American Heart Association Stroke Council. 2015 American Heart Association/American Stroke Association focused update of the 2013 guidelines for the early management of patients with acute ischemic stroke regarding endovascular treatment: a guideline for healthcare professionals from the American Heart Association/American Stroke Association. Stroke 2015;46(10):3020-3035

2 Mair G, Wardlaw JM. Imaging of acute stroke prior to treatment: current practice and evolving techniques. Br J Radiol 2014;87 (1040):20140216 
3 Saver JL, Gornbein J, Starkman S. Graphic reanalysis of the two NINDS-tPA trials confirms substantial treatment benefit. Stroke 2010;41(10):2381-2390

4 Rose JC, Mayer SA. Optimizing blood pressure in neurological emergencies. Neurocrit Care 2006;4(01):98

5 Jones TH, Morawetz RB, Crowell RM, et al. Thresholds of focal cerebral ischemia in awake monkeys. J Neurosurg 1981;54(06): 773-782

6 Hacke W, Donnan G, Fieschi C, et al; ATLANTIS Trials Investigators; ECASS Trials Investigators; NINDS rt-PA Study Group Investigators. Association of outcome with early stroke treatment: pooled analysis of ATLANTIS, ECASS, and NINDS rt-PA stroke trials. Lancet 2004;363(9411):768-774

7 Jauch EC, Saver JL, Adams HP Jr, et al; American Heart Association Stroke Council; Council on Cardiovascular Nursing; Council on Peripheral Vascular Disease; Council on Clinical Cardiology. Guidelines for the early management of patients with acute ischemic stroke: a guideline for healthcare professionals from the American Heart Association/American Stroke Association. Stroke 2013;44(03):870-947

8 Lansberg MG, Albers GW, Beaulieu C, Marks MP. Comparison of diffusion-weighted MRI and CT in acute stroke. Neurology 2000; 54(08):1557-1561

9 Thomalla G, Rossbach P, Rosenkranz M, et al. Negative fluidattenuated inversion recovery imaging identifies acute ischemic stroke at 3 hours or less. Ann Neurol 2009;65(06):724-732

10 Rimmele DL, Thomalla G. Wake-up stroke: clinical characteristics, imaging findings, and treatment option - an update. Front Neurol 2014;5:35

11 Barber PA, Demchuk AM, Zhang J, Buchan AM. Validity and reliability of a quantitative computed tomography score in predicting outcome of hyperacute stroke before thrombolytic therapy. ASPECTS study group. Alberta Stroke Programme Early CT Score. Lancet 2000;355(9216):1670-1674

12 Tarpley J, Franc D, Tansy AP, Liebeskind DS. Use of perfusion imaging and other imaging techniques to assess risks/benefits of acute stroke interventions. Curr Atheroscler Rep 2013;15 (07):336

13 Tomsick T, Brott T, Barsan W, et al. Prognostic value of the hyperdense middle cerebral artery sign and stroke scale score before ultraearly thrombolytic therapy. AJNR Am J Neuroradiol 1996;17(01):79-85

14 Tomsick T, Brott T, Barsan W, Broderick J, Haley EC, Spilker J. Thrombus localization with emergency cerebral CT. AJNR Am J Neuroradiol 1992;13(01):257-263

15 Bash S, Villablanca JP, Jahan R, et al. Intracranial vascular stenosis and occlusive disease: evaluation with CT angiography, MR angiography, and digital subtraction angiography. AJNR Am J Neuroradiol 2005;26(05):1012-1021

16 Josephson SA, Dillon WP, Smith WS. Incidence of contrast nephropathy from cerebral CT angiography and CT perfusion imaging. Neurology 2005;64(10):1805-1806
17 Krol AL, Dzialowski I, Roy J, et al. Incidence of radiocontrast nephropathy in patients undergoing acute stroke computed tomography angiography. Stroke 2007;38(08):2364-2366

18 Ang TE, Bivard A, Levi C, et al. Multi-modal CT in acute stroke: wait for a serum creatinine before giving intravenous contrast? No!. Int J Stroke 2015;10(07):1014-1017

19 Qureshi AI, Isa A, Cinnamon J, et al. Magnetic resonance angiography in patients with brain infarction. J Neuroimaging 1998;8(02):65-70

20 Muir KW, Buchan A, von Kummer R, Rother J, Baron JC. Imaging of acute stroke. Lancet Neurol 2006;5(09):755-768

21 Tansy AP, Liebeskind DS. Imaging acute ischemic stroke: mapping present and future clinical practice. Curr Atheroscler Rep 2015;17 (09):50

22 Albers GW. Endovascular Therapy Following Imaging Evaluation for Ischemic Stroke 3 (DEFUSE 3). Available at: https://clinicaltrials.gov/ct2/show $/$ NCT02586415?term $=$ DEFUSE $+3 \&$ rank $=1$. Accessed March 3, 2017

23 Albers GW, Thijs VN, Wechsler L, et al; DEFUSE Investigators. Magnetic resonance imaging profiles predict clinical response to early reperfusion: the diffusion and perfusion imaging evaluation for understanding stroke evolution (DEFUSE) study. Ann Neurol 2006;60(05):508-517

24 Lansberg MG, Straka M, Kemp S, et al; DEFUSE 2 study investigators. MRI profile and response to endovascular reperfusion after stroke (DEFUSE 2): a prospective cohort study. Lancet Neurol 2012;11(10):860-867

25 Amiri H, Bluhmki E, Bendszus M, et al. European Cooperative Acute Stroke Study-4: Extending the time for thrombolysis in emergency neurological deficits ECASS-4: ExTEND. Int J Stroke 2016;11(02):260-267

$26 \mathrm{Ma} \mathrm{H}$, Parsons MW, Christensen S, et al; EXTEND investigators. A multicentre, randomized, double-blinded, placebo-controlled Phase III study to investigate EXtending the time for Thrombolysis in Emergency Neurological Deficits (EXTEND). Int J Stroke 2012;7 (01):74-80

27 Copen WA, Rezai Gharai L, Barak ER, et al. Existence of the diffusion-perfusion mismatch within 24 hours after onset of acute stroke: dependence on proximal arterial occlusion. Radiology 2009;250(03):878-886

28 Turk A. POSITIVE: PerfusiOn Imaging Selection of Ischemic STroke Patlents for EndoVascular ThErapy. Available at: https://clinicaltrials.gov/ct2/show/study/NCT01852201. Accessed March 3, 2017

29 Jovin TG, Nogueira RG. Trevo and Medical Management Versus Medical Management Alone in Wake Up and Late Presenting Strokes (DAWN). Available at: https://clinicaltrials.gov/ct2/ show/NCT02142283. Accessed March 5, 2017

30 Deibler AR, Pollock JM, Kraft RA, Tan H, Burdette JH, Maldjian JA. Arterial spin-labeling in routine clinical practice, part 1: technique and artifacts. AJNR Am J Neuroradiol 2008;29(07):1228-1234

31 Paolini S, Burdine J, Verenes M, et al. Rapid short MRI sequence useful in eliminating stroke mimics among acute stroke patients considered for intravenous thrombolysis. J Neurol Disord 2013;1:137 